\title{
NATIONAL TRANSONIC FACILITY MODEL AND TUNNEL VIBRATIONS
}

\author{
John W. Edwards 1 \\ NASA Langley Research Center \\ Hampton, Virginia 23681-0001
}

\begin{abstract}
Since coming online in 1984, the National Transonic Facility (NTF) cryogenic wind tunnel at the NASA Langley Research Center has provided unique high Reynolds number testing capability. While turbulence levels in the tunnel, expressed in terms of percent dynamic pressure, are typical of other transonic wind tunnels, the significantly increased load levels utililized to achieve flight Reynolds numbers, in conjunction with the unique structural design requirements for cryogenic operation, have brought forward the issue of model and model support structure vibrations. This paper reports new experimental measurements documenting aerodynamic and structural dynamics processes involved in such vibrations experienced in the NTF. In particular, evidence of local unsteady airloads developed about the model support strut is shown and related to well documented acoustic features known as "Parker" modes. Two-dimensional unsteady viscous computations illustrate this model support structure loading mechanism.
\end{abstract}

\section{Introduction}

The National Transonic Facility (NTF) provides flight Reynolds number wind tunnel testing capability using gaseous nitrogen as a test medium at cryogenic temperatures at pressures up to 8.8 atmospheres ${ }^{1}$ Cryogenic temperatures are obtained by spraying liquid Nitrogen into the tunnel circuit. While the maximum design dynamic pressure is 7000 pounds per square foot (psf.), flight Reynolds numbers are achieved for most commercial aircraft models at dynamic pressures of about 3000 psf. Measurements of tunnel turbulence or unsteadiness, as typified by Figure 1 from Ref. 2, indicate that the NTF is typical of slotted wall transonic tunnels, with maximum perturbation mean square pressures of about one percent of dynamic pressure. Still, the very high dynamic load levels, coupled with the unique structural design and construction requirements for cryogenic operations, has brought forward the issue of model and structural vibrations.

\footnotetext{
1 Senior Research Engineer, Aeroelasticity Branch,
} Structures Division

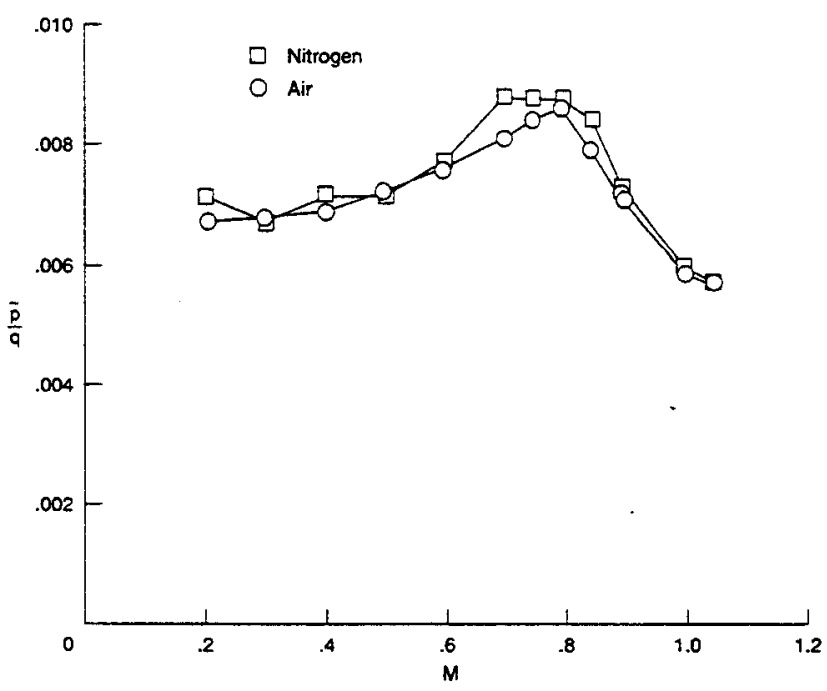

Figure 1 Fluctuating presure coefficient measured at test section right hand sidewall station $13 . \operatorname{Re}=6 \times 10^{6}$; ambient temperature; air and nitrogen modes. (from Ref. 2)

The purpose of this paper is to present details of a novel aeroacoustic mechanism which plays a role in the model vibrations. Experience with model vibrations and measurements of tunnel unsteadiness are briefly summarized. Then the related topics of wind tunnel "resonance" from the aeroelastic literature and "Parker" modes from the aeroacoustic literature are discussed. NTF measurements are then shown indicating the presence of Parker mode interaction with strut dynamics during the occurrence of model vibrations under certain test conditions.

\section{Background}

Young et al. ${ }^{3}$ and Buerhle et al. ${ }^{4}$ give details of model vibrations experienced in the NTF, focusing upon model yawing oscillations about the model support structure, shown in Figure 2. The model, balance, and sting are connected to the model support structure, herein termed the "strut", which consists of the moveable arc sector quadrant, (the forward half of the strut), and the aft aerodynamic body, termed the "fixed fairing". The strut has a chord, c, of $9.2 \mathrm{ft}$. and the dimensions of the test section are $8.2 \mathrm{ft}$. by $8.2 \mathrm{ft}$. Figure 3 shows the cross section profile of the strut and the wind tunnel walls in the 
vicinity of the strut. The trailing edge of the fixed-fairing has a $40 \mathrm{deg}$. included angle. A slightly longer fairing would have allowed a smaller trailing edge angle but was precluded due to clearance for the plenum-test section isolation "gate-valve" mechanism at the downstream plenum bulkhead. The walls diverge to allow "area ruling" of the strut thickness and aft of the trailing edge the transition from the square test section cross section to the conical diffuser section begins, with wall divergence half-angle of approximately $2.1 \mathrm{deg}$.

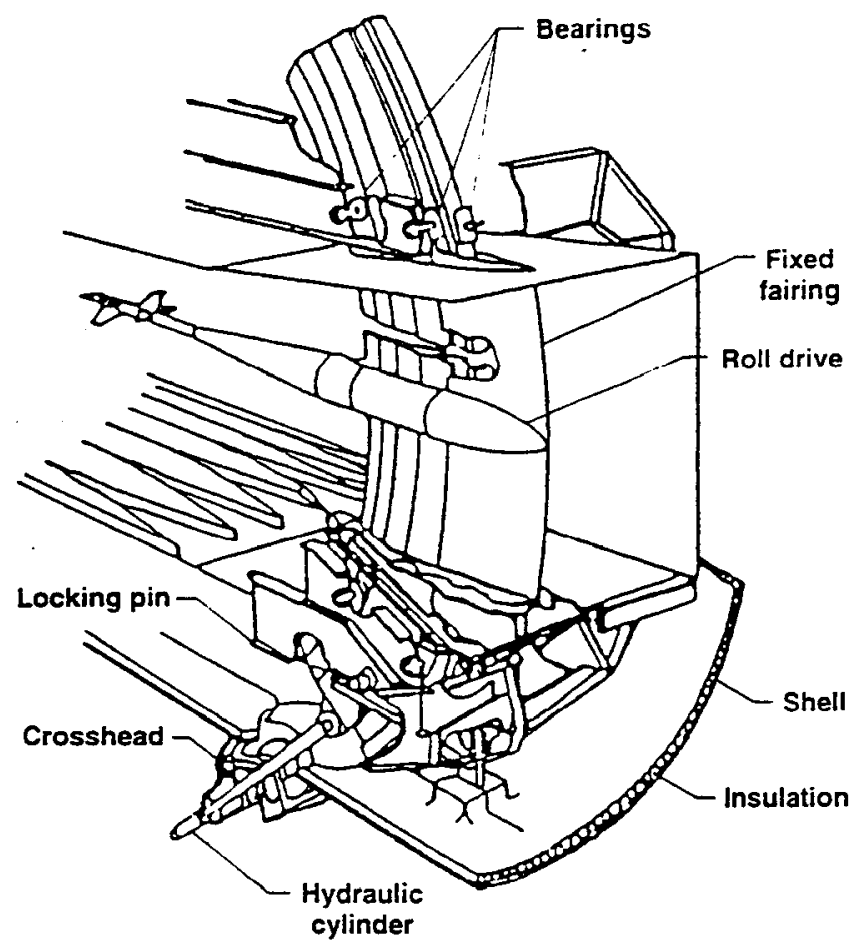

Figure 2 NTF model support system. (from Ref. 3)

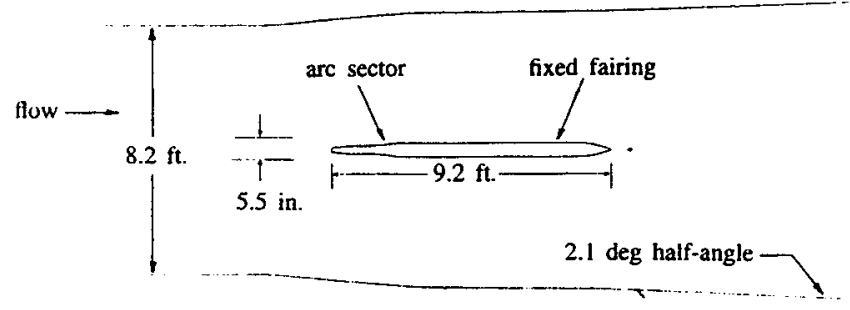

Figure 3 Cross section along tunnel centerline of NTF strut (arc sector plus fixed fairing) and tunnel sidewalls.

The arc sector is restrained laterally by four pairs of "pucks", two at the top and two at the bottom. These pucks were originally designed for a 0.010 in. clearance ${ }^{3}$.
Adjustment of the pucks was troublesome and excessive clearance was shown to be detrimental for model vibrations. This has led to a modified puck design wherein positive contact between pucks and the arc sector is maintained using preloaded Belleville washers under the puck attachment bolts.

Vibration measurements performed on modelbalance-sting-strut combinations ${ }^{4}$ have provided insight into the dynamics involved in the model vibrations. Frequencies vary with each particular combination tested and typically produce sting bending modes somewhat below $10 \mathrm{~Hz}$.; combination sting bending with model yawing about the balance somewhat above $10 \mathrm{~Hz}$.; and three modes involving the model support strut. For the combination studied in Ref. 3 these modes occurred at 19.2, 23.8 and $29.7 \mathrm{~Hz}$. These strut modes result from the design requirements to accommodate thermal expansion/contraction of the tunnel structures. The fixed fairing is rigidly attached to the test section backup structure at the top, but is pinned at the bottom. This, plus the flexibility of the arc sector restraints, gives rise to the vibration modes. The $29.7 \mathrm{~Hz}$. mode shape is indicated in Figure 4 and is described as "arc sector/fixed fairing rotation with model yaw on balance".

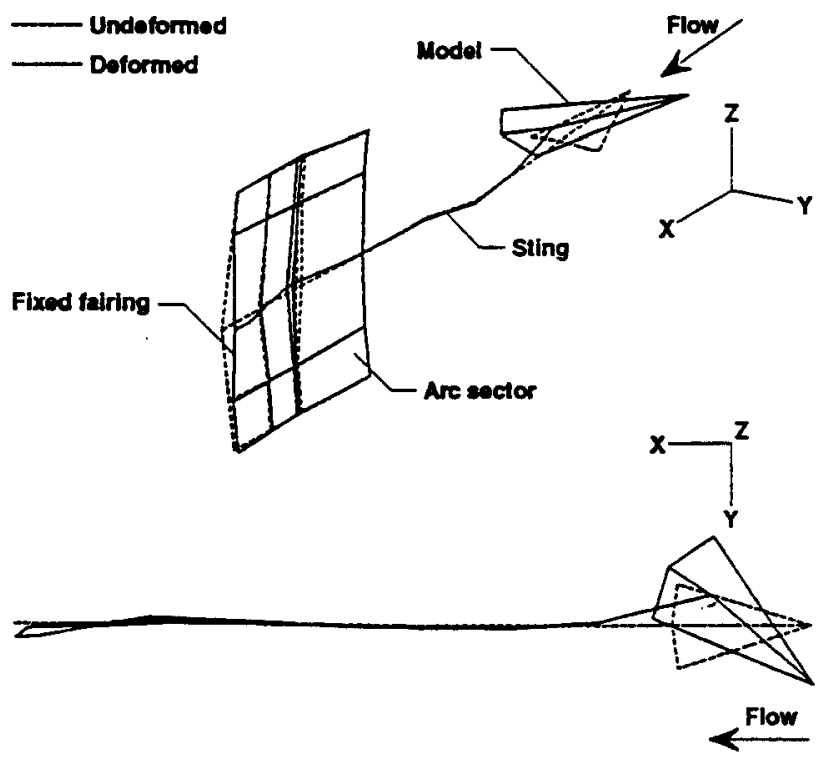

Figure 4 Model support strut, sting, balance, and model mode shape for $29.7 \mathrm{~Hz}$. vibration mode. (from Ref. 4)

Young et al. ${ }^{5}$ present details of another type of model vibration in the NTF; rolling oscillations of a Boeing 767 model about the roll balance. This case did not involve other components of the model support structure and was shown to be induced by buffet onset of the model on the sting. Also documented was the elimination of the rolling oscillations when scaled replicas of the vortex generators 
installed on production aircraft were tested on the model. Particularly noteworthy was the sensitivity of the results to Reynolds number, emphasizing the importance of wind tunnel tests at flight Reynolds number for such buffet onset testing.

Igoe $^{2}$ made dynamic measurements of fluctuating pressure levels at 11 locations in the NTF circuit across the complete tunnel operating range and many specific conclusions regarding the dynamic behavior of the tunnel are given. Most dynamic pressure spectra shown cover a wide frequency range, $0-20 \mathrm{kHz}$., and do not focus on the $0-50 \mathrm{~Hz}$. range where model vibrations occur.

Following early evidence of model vibrations, two aeroelastic analyses were made of the NTF strut. Strganac ${ }^{6}$ performed flutter analyses for Mach number, $M=0.5$, using doublet lattice linear theory aerodynamics and found a tendency for a damping dependent "hump" mode instability within the operating envelope of the tunnel. Small levels of damping, always present in builtup structures, would eliminate such analytical instabilities, but the resulting lightly damped mode(s) are susceptible to disturbances and can produce high response levels. The wind tunnel walls were not modeled in this study.

Whitlow et al. ${ }^{7}$ performed similar aeroelastic analyses, using the inviscid CAP-TSD (Computational Aeroelasticity Program - Transonic Small Disturbance) code. The analysis, for several subsonic Mach numbers, again showed hump mode instabilities for zero assumed damping. With the wind tunnel walls modeled as parallel solid walls, the analysis showed the presence of an additional modal response which was attributed to wall interference and which interacted with the structural modes involved in the hump mode instabilities. The interaction was strongest for intermediate subsonic Mach numbers leading to the observation that the mechanicsm might not be observed at higher speed design conditions.. The discussion of the additional mode in Ref 7. identifies it as the wind tunnel resonance frequency introduced by Runyan et al. ${ }^{8}$. It will be shown below that this mode is instead a local acoustic mode studied extensively by Parker ${ }^{9-11}$ and termed the $\beta$-mode.

\section{Wind Tunnel "Resonance"}

Runyan et al. ${ }^{8}$ studied the problem of an airfoil in a solid wall subsonic wind tunnel undergoing forced pitching oscillations. The tunnel height to airfoil chord ratio was 3.6:1. Measurements had shown that the lift and moment on the airfoil went to zero at a frequency dependent upon Mach number. Subsonic integral equation lifting surface theory was developed for the airfoil between solid, reflecting walls and a numerical solution procedure developed. It was noted that the kernel of the integral equation relating lifting pressure to the downwash bound- ary condition became infinite at frequencies

$$
f_{r e s}=\frac{a_{\infty} \sqrt{1-M^{2}}}{2 h}(2 n-1), n=1,2,3, \ldots
$$

where $a_{\infty}$ is the freestream sound speed and $h$ is the tunnel height. Since the integrated product of the kernel and lifting pressure over the airfoil must equal the finite vertical velocity, the lifting pressure must become zero at these frequencies. The lowest frequency in this series, for $n=1$ has come to be termed the tunnel resonance frequency, $f_{\text {res }}$. Calculations were accomplished for $f \leq f_{\text {res }}$ and a typical comparison with experiment is shown in Figure $5^{8}$. The lift ratio is the ratio of lift for

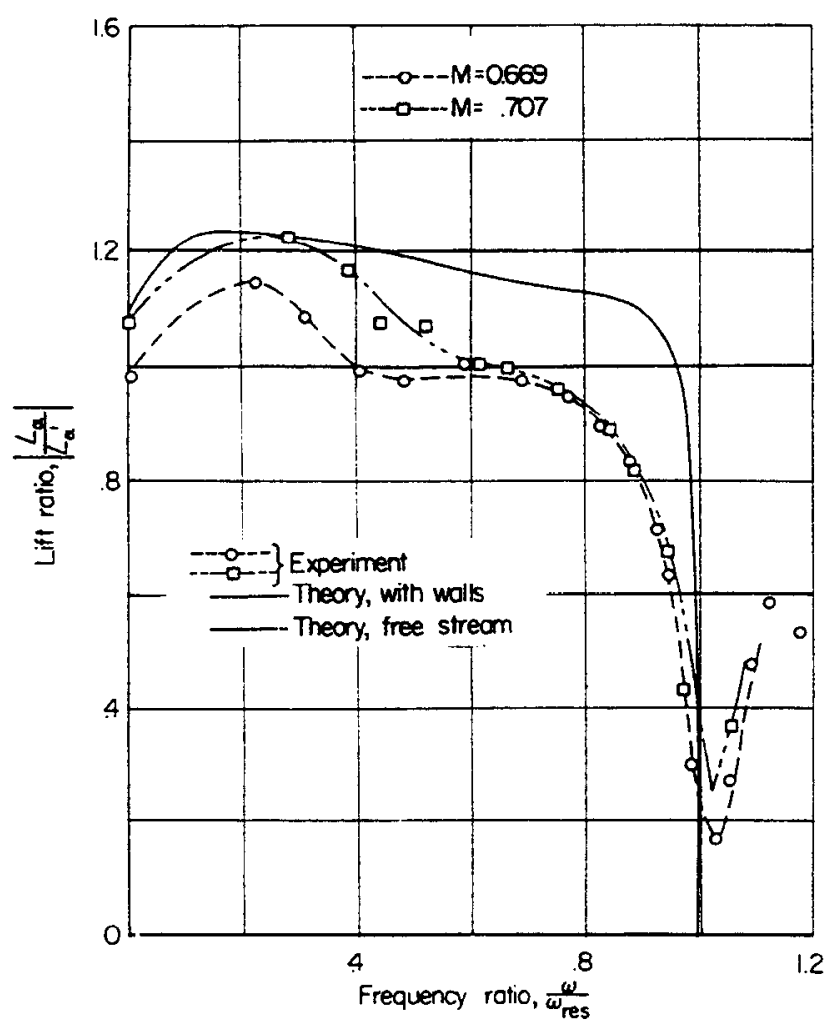

Figure 5 Ratio of magnitude of lift in wind tunnel to lift in free air for a pitching airfoil with $h / c=3.6$. (from Ref. 8)

the airfoil in the tunnel to the lift in free air. Most of the discussion in the ensuing aeroelastic literature of wind tunnel wall interference in unsteady testing has focused mainly on this $f_{\text {res }}$ eigenmode. It should be noted that this is an eigenmode of the empty wind tunnel, as the development of no lift can be seen as the result of the airfoil "following" the local vertical motion of the fluid oscillation in an undamped eigenmode. Thus the fluid is unaware of the presence of the body and the same eigenmode obtained with the body removed. This resonance frequency is the same as the $\omega_{3}$ eigenmode of Lee ${ }^{13}$ who studied resonanant frequencies in open 
and closed three dimensional empty wind tunnels using the finite element method. Mabey ${ }^{13}$ further developed the resonance conditions for empty ventilated (slotted or porous) tunnels with plenum chambers. Resonance frequencies are shown to be below those of the closed tunnel for $M \leq 0.618$ and greater for $0.618 \leq M \leq 1.0$.

In this unsteady aerodynamic literature, the focus has been on undamped eigenmodes of the empty tunnel. In terms of the lift ratio shown in Figure 5, this appears as an antiresonance. Attention is drawn to the damped resonance feature seen in Figure 5 near $f \approx 0.2 f_{\text {res }}$ which has not been preiviously discussed. This is shown below to be the $\beta$-mode of Parker?

\section{“Parker” Acoustic Modes}

In a series of publications, Parker has studied acoustic resonances excited between cascades of parallel flat plates wherein the resonances are excited by vortex shedding from the blunted trailing edges. Ref. 9 provided

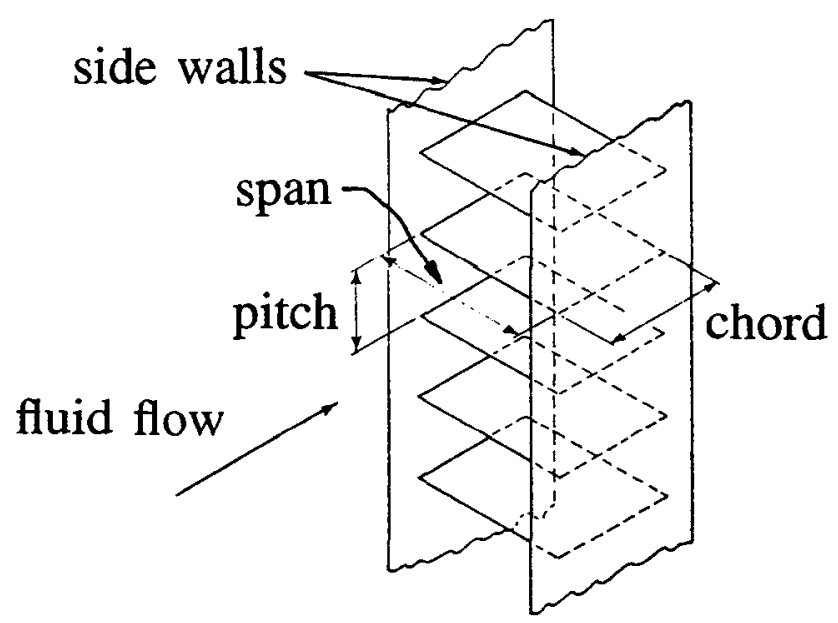

Figure 6 Parker's experimental apparatus for investigating acoustic modes about parallel flat plates. (from Ref. 9)

early calculations of the resonances and Ref. 11 is a recent summary article. Figure 6, from Ref. 9, indicates the typical configuration studied in a low speed wind tunnel. Due to the repetitive geometry of the cascade, it was recognized that one cell of the cascade can be representative of a plate (or an airfoil or model support strut) between solid wind tunnel walls. The acoustic modes observed by Parker are identified as local, nonradiating modes with frequencies below the empty duct "cutoff" frequency. The four most prominent, lower frequency modes are termed the $\beta, \alpha, \delta, \gamma$ modes. Figure 7, from Kock $^{14}$ displays these calculated mode frequencies as a "Parker mode diagram" for $\mathrm{M}=0$ and a cascade with zero stagger angle. The notation $(m, n)$, commonly used for cavity modes, is employed to order the resonant modes. Here $m$ denotes the number of nodal lines of the pressure between the leading and trailing edge of the cascade and $n$ denotes the number of nodal lines between two plates. ${ }^{14}$ The shading in the inserts indicates the relative pressure levels between plates for the four prominent modes. The circles in the figure represent Parker's experimental results while the crosses stand for Parker's numerical solutions. ${ }^{9}$ The abscissa, $f_{*}=f h / a_{\infty}=f / 2 f_{\text {res }}$ at $M=0$ and it should be noted that, as the chord $\mathrm{c}$ goes to zero, the $\beta$-mode frequency approaches $f_{*}=0.5$ and $f=f_{\text {res }}$. That is, the $\beta$-mode becomes the empty tunnel wind tunnel resonance mode of Runyan ${ }^{8}$ as the chord shrinks to zero.

All of these modes are acoustically nonpropagating and decay exponentially away from the airfoil. In the immediate vicinity of the plates pressure fluctuations on the order of the dynamic pressure can be observed. Parker ${ }^{11}$ comments that the resonant frequencies decrease with increasing Mach number in proportion to $\left(1-M^{2}\right)^{n}$, where $n$ depends on the ratio $c / h$ and is in the range $0.5<n<1.0$. Note that the Mach number dependence of Runyan's wind tunnel resonance mode, $f_{\text {res }}$ is given by this relatation with $n=0.5$ which will be assumed in the following. Koch's ${ }^{14}$ Figure 5. demonstrates this trend for the $\beta$-mode, also showing that the modal damping ratio increases from $\zeta=0$ for $M=0$ to $\zeta \approx 0.25$ for $M=0.7$. For the NTF strut, $c / h \approx 9.2 / 8.2=1.12$ and, from Figure $7, f_{*} \approx 0.32$ for the $\beta$-mode. Thus, for the NTF strut

$$
f_{\beta}=0.32\left(a_{\infty} / 8.2\right) \sqrt{1-M^{2}}
$$

A good approximation for the speed of sound in both air and nitrogen is $a_{\infty}=49 \sqrt{T,{ }^{\circ} \mathrm{F}+460}, \mathrm{ft} / \mathrm{sec}$.

Parker also investigated the effect of the $\beta$-mode interacting with a flexible steel plate in a channel ${ }^{10}$, a study which is interestingly similar to the NTF strut. By varying the channel width, the $\beta$-mode frequency was able to be placed below, at, and above the fundamental plate bending frequency. Very interesting details of the energy exchange between the airflow and the structural plate are discussed. Ref. 11 summarizes these results, recommending that the situation wherein $f_{\beta}$ is slightly below the plate natural frequency is to be avoided since it produces the largest structural response levels. 


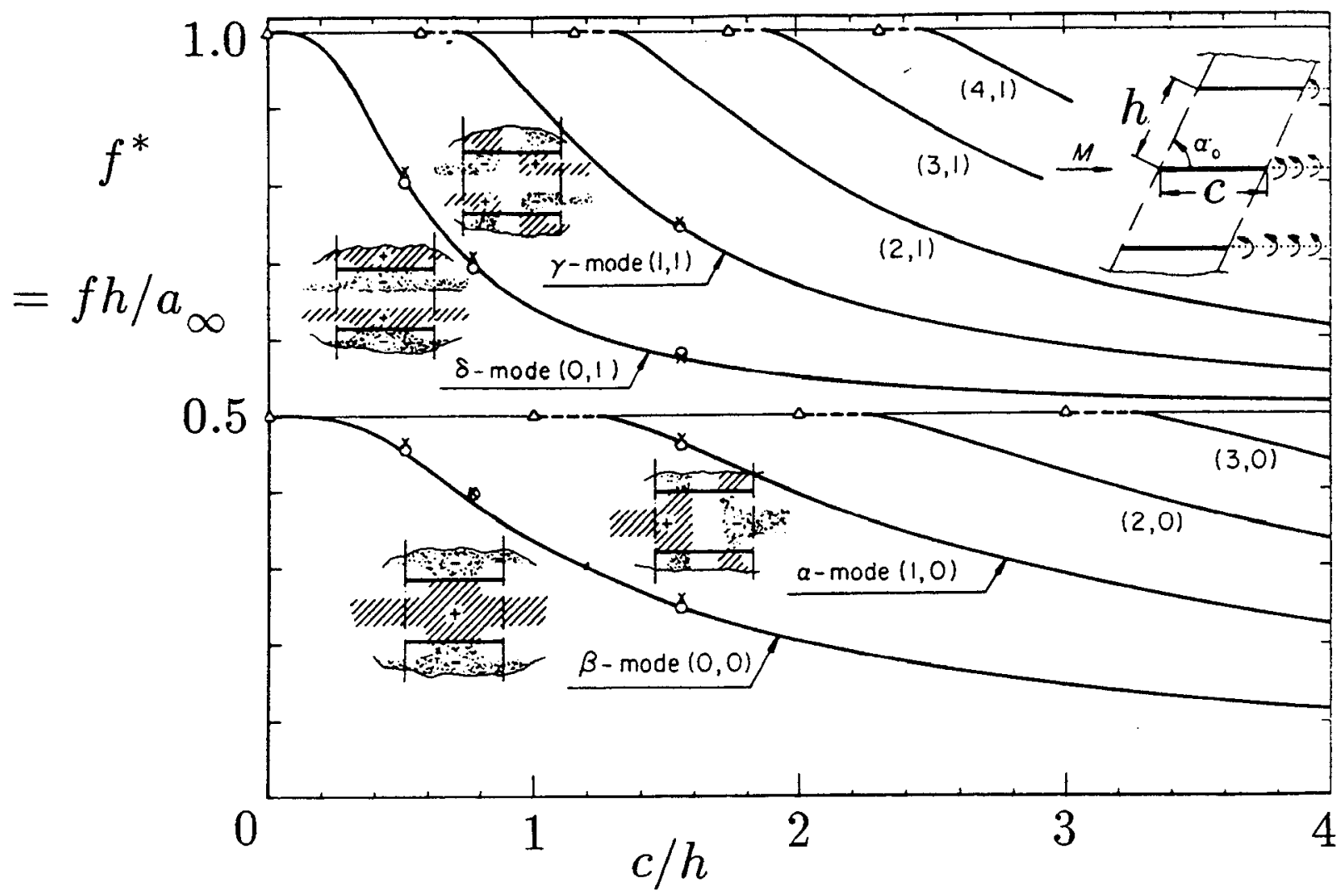

Figure 7 "Parker mode diagram" for $M=0$ and $\alpha_{0}=\pi / 2$. $O$, Parker experiment; $\times$, Parker computation. (from Ref. 14)

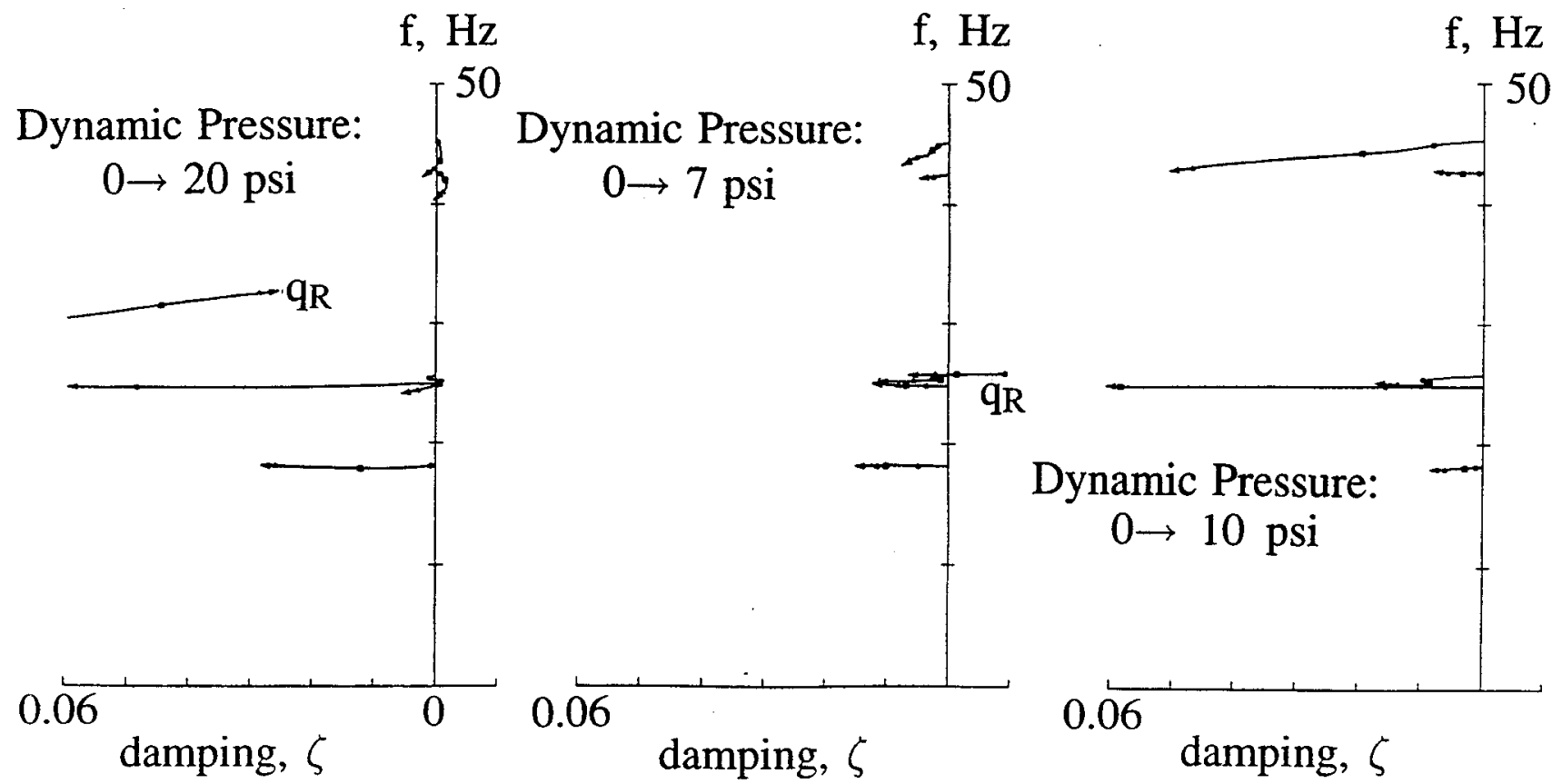

Figure 8 Aeroelastic root loci of strut modes and $\beta$-mode for varying Mach number and dynamic prressure calculated with CAP-TSD code. (from Ref. 7 ) 
Analysis

In this section several efforts to analyze details of the aerodynamic and aeroelastic behavior of the strut are discussed. These efforts preceeded the experimental measurements in the NTF to be discussed in the following sections.

First, results from the aeroelastic analysis of the strut by Whitlow et al. ${ }^{7}$ using the inviscid CAP-TSD code are summarized in Figure 8. Five elastic mode shapes, derived from vibration testing of the strut, were used in the analysis. Transient response time histories of simulated strut motions were analyzed to extract frequencies and dampings. Results for $\mathrm{M}=0.43,0.6$, and 0.8 and for varying dynamic pressures. were computed. Calculations were made for the strut in "free air" (large computational grid) and for the strut between the NTF walls, modeled as reflecting, parallel upper and lower computational grid boundaries. The results shown in Figure 8 are with the walls modeled. Positive damping ratios, $\zeta$, are stable and the results show "hump" mode instabilities for $\mathrm{M}=0.43$ and 0.6. That is, small regions of negative damping are seen for limited ranges of dynamic pressure. Since the analysis is performed for zero assumed damping, realistic damping levels would be expected to provide stability in reality.
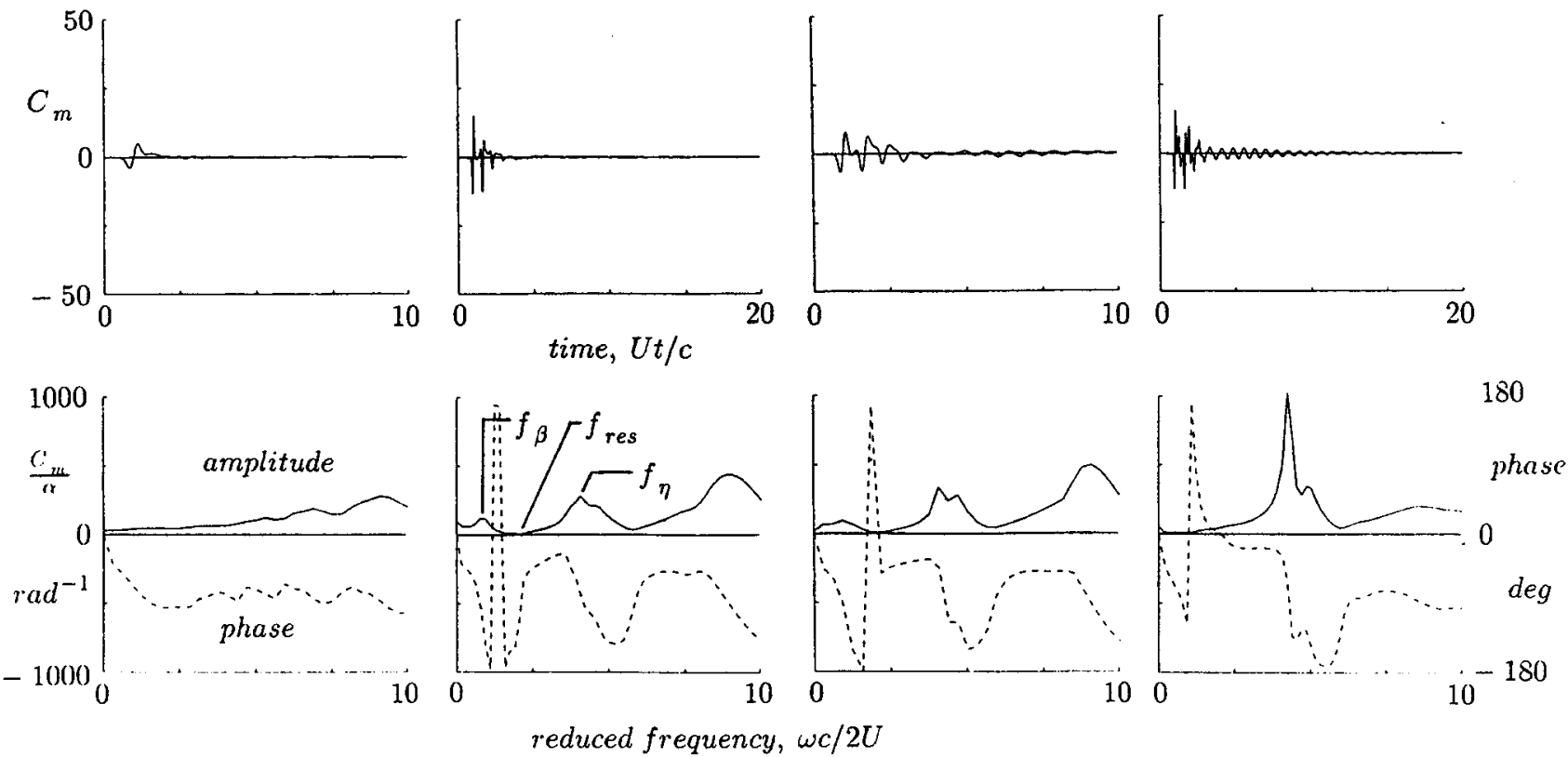

reduced frequency, $\omega c / 2 U$

a.) linear equation flat plate geometry free air solution

The figure also indicates that for the two lower Mach numbers an additional mode, labeled $q_{r}$, was identified near $25-33 \mathrm{~Hz}$. Now Eq. 2 predicts that the frequency of the $\beta$-mode for $\mathrm{T}=+120^{\circ} \mathrm{F}$ and for $\mathrm{M}=0.43$ and 0.6 is $f_{\beta}=41.5$ and $36.8 \mathrm{~Hz}$. respectively. It is now evident that the $q$, mode is the $\beta$-mode interacting with the strut modes to produce the hump mode instabilities shown in the figure.

The second analysis used a two-dimensional version of the inviscid CAP-TSD code to study effects of flow equation level and strut and wall geometry modeling on the behavior of the strut unsteady airloads. Simulated pulsed motions of the strut pitching about its midchord were computed and the resulting airloads analyzed to obtain frequency response functions. Figure 9 gives results for $\mathrm{M}=0.65$ for the pitching moment coefficient versus reduced frequency, $k=\omega c / 2 U_{\infty}$. In the free air solution no resonances are seen, whereas pronounced resonances are seen for the three cases with walls modeled. In Figure $9 \mathrm{~b}$., the $\beta$-mode is seen at $k=0.85$ which corresponds to $f_{\beta}=33.8 \mathrm{~Hz}$. for the NTF strut. Also seen is the antiresonance, $f_{\text {res }}$, where the amplitude of the airloads drops to zero. Finally, two additional prominent resonances at higher frequencies are noted. The first of these, labeled here $f_{\eta}$, occurs at approximately $5 f_{\beta}$ and would appear to be a higher order Parker mode than those shown in Figure 7.
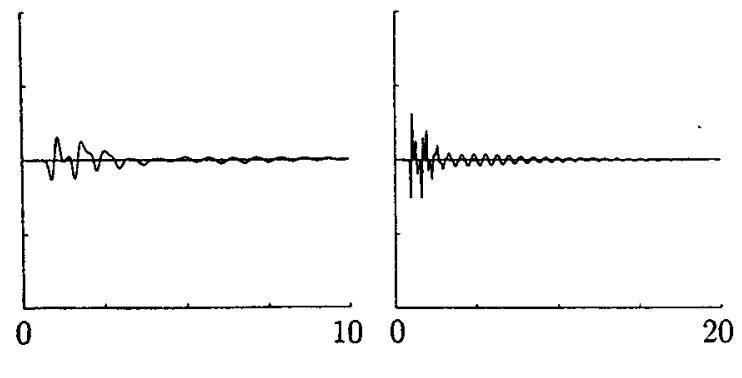
b.) linear equation flat plate geometry parallel walls

d.) TSD equation strut geometry NTF diverging walls

Figure 9 Frequency responses for strut pitching moment due to pitching calculated with CAP-TSD code: $\mathrm{M}=0.65$, ambient temperature. 
Figure 9c. indicates that these features are unchanged with the introduction of the strut geometry and the TSD equation. On the other hand, introducing the wall divergence, Figure 9d., affects the three features discussed above: the $f_{\beta}$ and $f_{\text {res }}$ features are no longer seen; and the $f_{\eta}$ resonance is much enhanced. This is indicative of selective "tuning" of these local acoustic modes through small changes of body and wall boundary geometries. Calculations, not shown, with a viscous version of the 2-D CAP-TSD code ${ }^{15,16}$, using an interactive boundary layer method, show dominant $\beta$-mode activity for the conditions of Figure 9d. This is due to the slight, though critical, change to the effective strut geometry introduced by the boundary layer displacement thickness.

The final analysis to be discussed is for the conditons of Figure 9d. Calculations were made with two thin layer Navier-Stokes (TLNS) codes to study the fluid dynamic behavior of the flow about the strut. These codes are described in Ref. 17 where their use in calculating transonic shock-induced oscillations about airfoils is shown. Figure 10 gives transient responses calculated with the CFL3D and TLNS3D codes. Perturbations were introduced and the lift coefficient responses, with $t^{*}=a_{\infty} t / c$, show damped $\beta$-mode response at $27 \mathrm{~Hz}$. with $\zeta \approx 0.03-0.05$. These calculations and the above viscous TSD calculations indicate a marked sensitivity to the detailed viscous flow at the strut trailing edge, which has a 40 degree included angle closure. Both types of flow modeling indicated a small region of flow separation at the trailing edge, on the order of several percent chordlength. But the viscous TSD calculations showed large self-excited $\beta$-mode flow oscillations, whereas the TLNS results are damped.

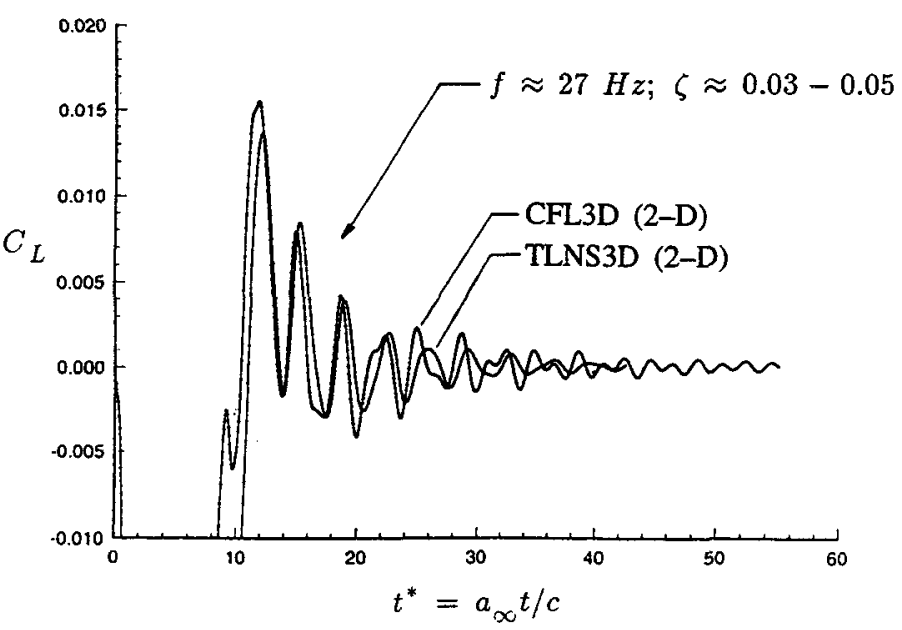

Figure 10 Strut lift coefficient transient reponses calculated with thin layer Navier-Stokes codes: $\mathrm{M}=0.65$, ambient temperature.

\section{Instrumentation}

Instrumentation installed to study the behavior of the strut is indicated in Figure 11. Eight unsteady pressure transducers were placed on the tunnel side walls along the tunnel centerline at approximately $0,33,67$, and 100 percent chord. On the far side wall, the leading sensor was placed approximately 33 percent chord ahead of the strut leading edge. Two additional unsteady pressure sensors were installed on opposite sides of the fixed fairing, near the aft end of the flat section of the fairing. At the same locations, two accelerometers were placed to measure the strut (fairing) lateral and vertical accelerations. Finally, two strain gage measurements were obtained from the model balance, one indicative of pitching moment and the other of yawing moment. Due to concern over tunnel data system integrity, these single strain gage measurements are uncalibrated and only relative amplitudes can be given. The measurements shown below are from this "yaw" signal, the PW7 side wall pressure sensor, and the AS1 vertical strut accelerometer.

$$
\begin{array}{ll}
\text { P - unsteady pressure sensor } & \text { S - strut } \\
\text { A - accelerometer } & W \text { - wall }
\end{array}
$$

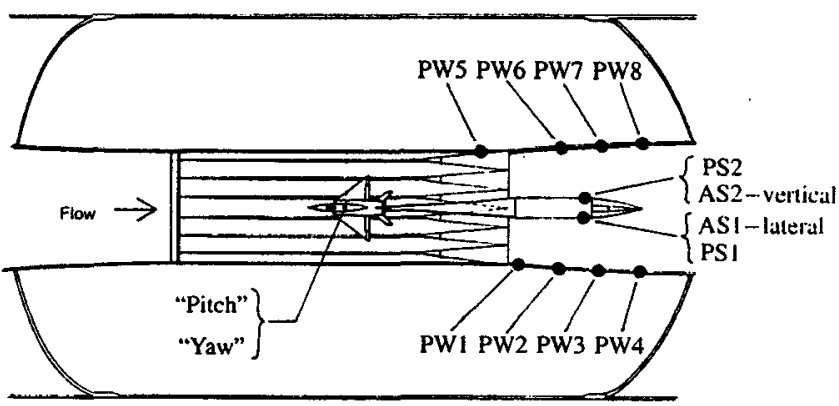

Figure 11 Instrumentation for the present investigation.

The model installed in the tunnel was the High Speed Research model described in Ref. 3. Data was recorded for playback analysis on a 28 channel analog tape recorder. Spectral analysis was accomplished with a Spectral Dynamics analyzer with 4 channel input capability. Frequency spectra were generally analyzed for a $0-100 \mathrm{~Hz}$. bandwidth and analog low pass prefilters were used.

For these measurements, data was recorded from the time of the start of the tunnel drive fan until the tunnel had achieved test conditions at $M=0.9$, generally about 10-30 minutes. The results shown below were obtained with one hundred spectral averages and, due to the changing tunnel conditions, some smearing of spectral features should be expected. 
$M \sim 0.20 \quad Q \sim 88$ psf.

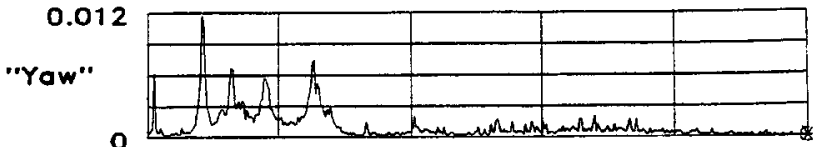

0.10

AS1, g's

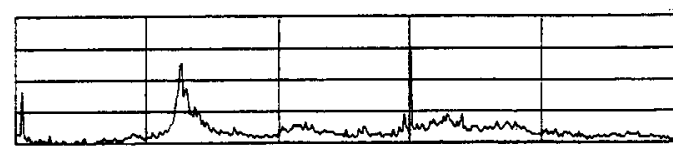

0.0002

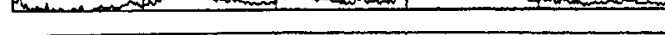

PW7.psi.

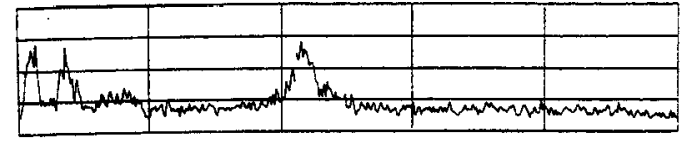

$M \sim 0.40 Q \sim 300$ psf.
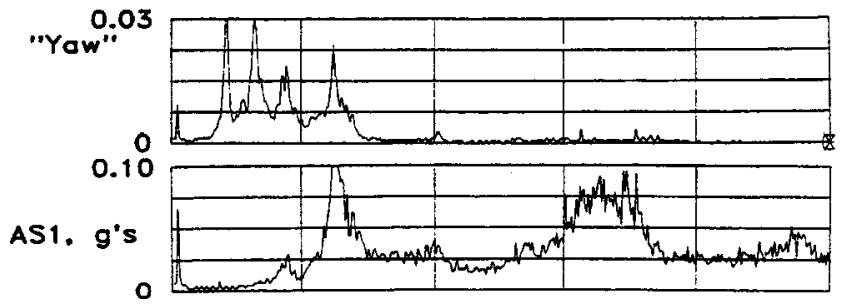

0.001

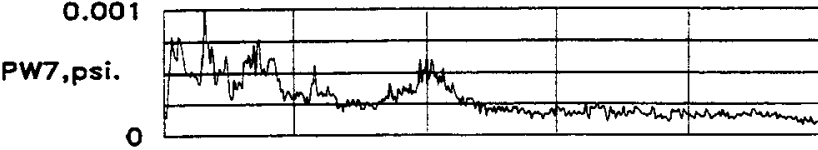

$M \sim 0.60 \quad Q \sim 584$ psf.

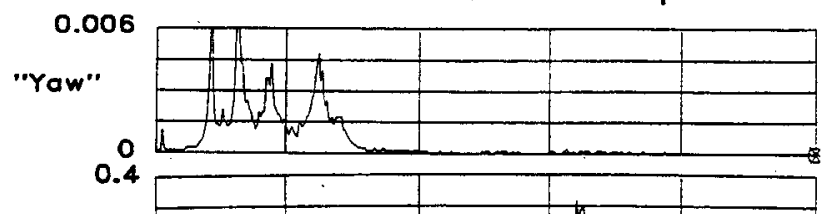

AS1, g's

0.002

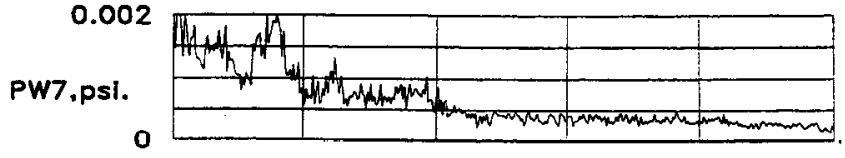

$M=0.90 \quad Q=965$ psf.

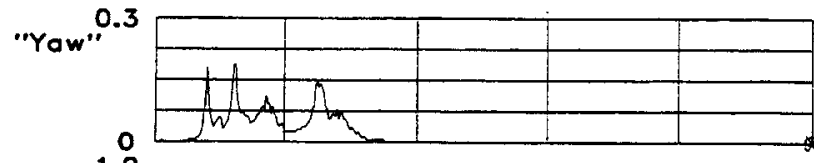

A51. g's

.0

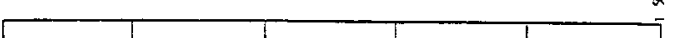

0.005

PW7,psi.
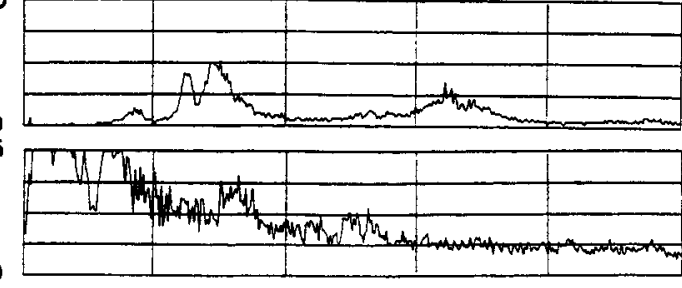

f. $\mathrm{Hz}$.

100

a.) $T=+120 \operatorname{deg} F$.
$M \sim 0.20 Q \sim 140$ psf.

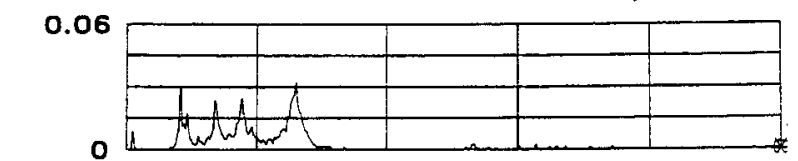

0.0004

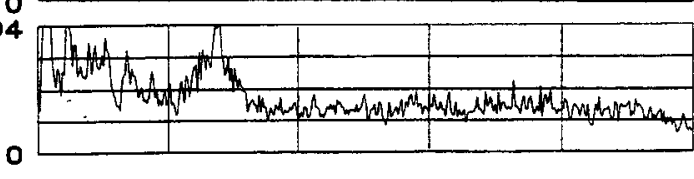

$$
M \sim 0.40 \quad Q \sim 450 \text { psf. }
$$
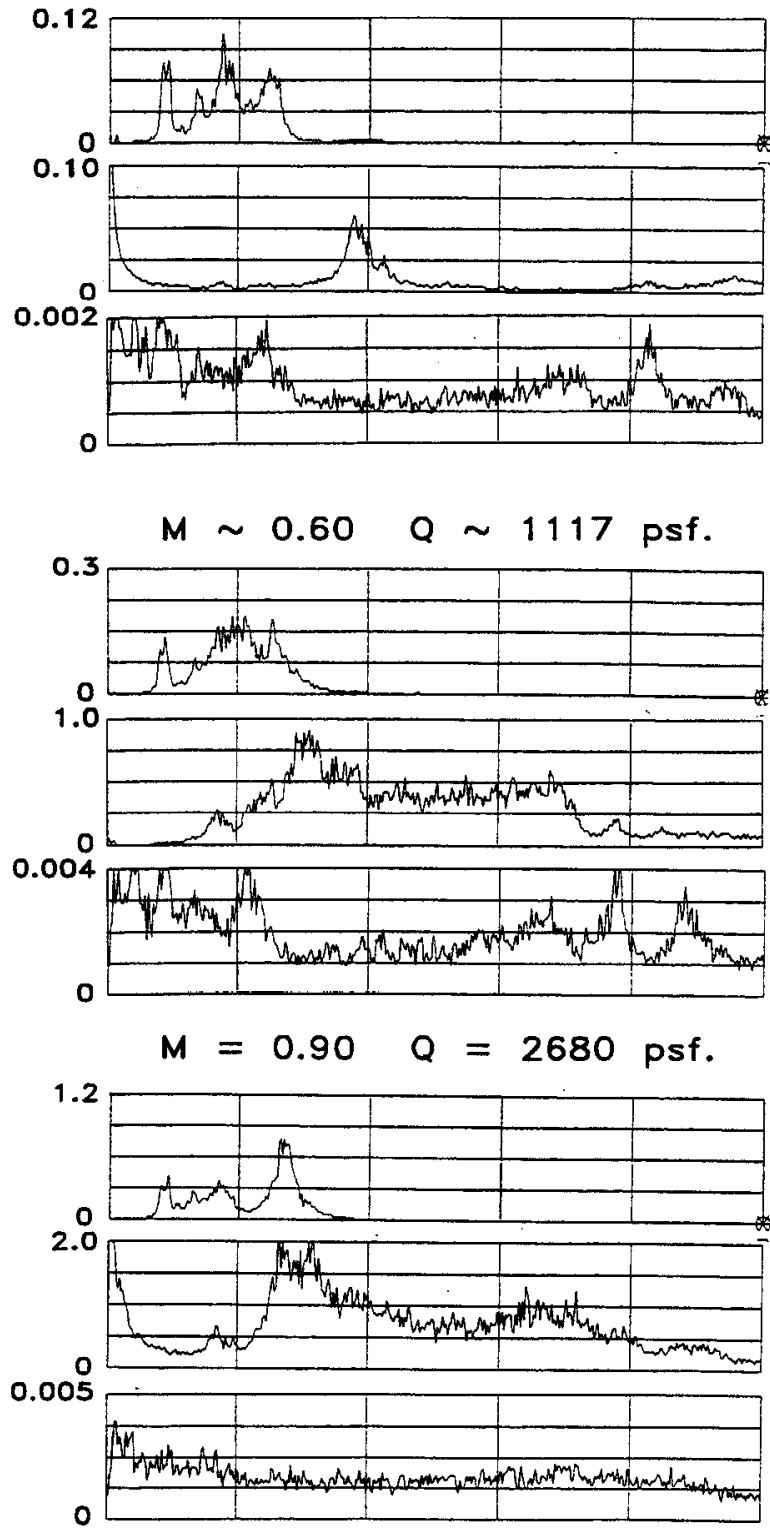

0

f, $\mathrm{Hz}$.

b.) $T=-250 \operatorname{deg} F$.

Figure 12 Frequency spectra from High Speed Research Model test. 


\section{Results}

Frequency spectra from the three sensors described above are shown in Figure 12 for $\mathrm{T}=+120^{\circ} \mathrm{F}$ and $-250^{\circ} \mathrm{F}$ and for $\mathrm{M}=0.2,0.4,0.6$, and 0.9 . This results in dynamic pressures in the test section of 965 psf. for the ambient temperature and 2680 psf. for the cryogenic tempertature at $\mathrm{M}=0.9$. Attention is drawn to the behavior of the spectra in the $20-40 \mathrm{~Hz}$. frequency range, as it is this range where the largest model vibrations are observed. The "yaw" spectra typically contain several resonance peaks below $20 \mathrm{~Hz}$. which are associated with sting and model/balance/sting/strut dynamics. This model/balance/sting combination also has a resonance peak in the range of $25-28 \mathrm{~Hz}$. which is in close proximity to the resonance usually seen in the strut lateral acceleration, AS1, spectra.

The side wall pressure spectra from the PW7 sensor show spectral features below $20 \mathrm{~Hz}$. which maintain the same general shape for the differing conditions, rising in amplitude with increasing Mach number and dynamic pressure. The key feature to be noted are the spectral peaks seen near $44 \mathrm{~Hz}$. for $\mathrm{T}=+120^{\circ}$ and $\mathrm{M}=0.2$ and near $27 \mathrm{~Hz}$. for $\mathrm{T}=-250^{\circ}$ and $\mathrm{M}=0.2$. These resonances decrease in frequency with increasing Mach number for both temperatures, becoming difficult to distinquish for the higher Mach numbers. This resonance is that of the Parker $\beta$-mode as can be seen from the agreement of these measured frequencies with those predicted by Eq. 2 in Table I. The agreement is quite good for the lower Mach numbers where the mode is distinct. The difficulty in discerning the mode at the higher Mach numbers is consistent with the increased damping at higher Mach numbers discussed above. Finally, note that the 0.004 psi. spectral amplitude seen at $f \approx 23 \mathrm{~Hz}$. for $\mathrm{M}=$ 0.6 and $\mathrm{T}=-250^{\circ}$ would be representative of a 40 pound force per side pressing on the strut at this frequency, assuming that this is a coherent pressure loading over the entire strut surface. The other pressure sensors, including the two on the strut, show spectral features similar to those shown in Figure 12.

Table 1 Frequency of $\beta$-mode versus Mach number and temperature for the NTF model support strut.

\begin{tabular}{|l|l|l|l|l|}
\hline & \multicolumn{2}{|c|}{$\mathrm{T}=+120^{\circ} \mathrm{F}$} & \multicolumn{2}{c|}{$\mathrm{T}=-250^{\circ} \mathrm{F}$} \\
\hline $\mathrm{M}$ & \multicolumn{2}{|c|}{$f, \mathrm{~Hz}}$. & \multicolumn{2}{c|}{$f, \mathrm{~Hz}}$. \\
\hline & Eq. 2 & exp. & Eq. 2 & exp. \\
\hline 0.0 & 46.0 & - & 27.7 & - \\
\hline 0.2 & 45.0 & 43.0 & 27.2 & 28.0 \\
\hline 0.6 & 36.8 & -37.0 & 22.2 & 23.0 \\
\hline 0.8 & 27.6 & - & 16.6 & - \\
\hline 0.9 & 20.1 & -33.0 & 12.1 & - \\
\hline
\end{tabular}

The relative amplitude of the "yaw" spectral peak at $25-28 \mathrm{~Hz}$. is indicative of the amplitude of model vibrations. Figure 13 summarizes this feature for the High Speed Resarch model. Data from test runs at three additional temperatures are given. The test condition for each run was $\mathbf{M}=0.9$ and differing tunnel static pressures lead to differing maximum dynamic pressures which in turn is indicative of the maximum power in the flow within the tunnel. It should be expected that the amplitude of model vibration would increase with increasing dynamic pressure, assuming broadband non-resonant turbulence as a loading mechanism. This generally rising level of "yaw"

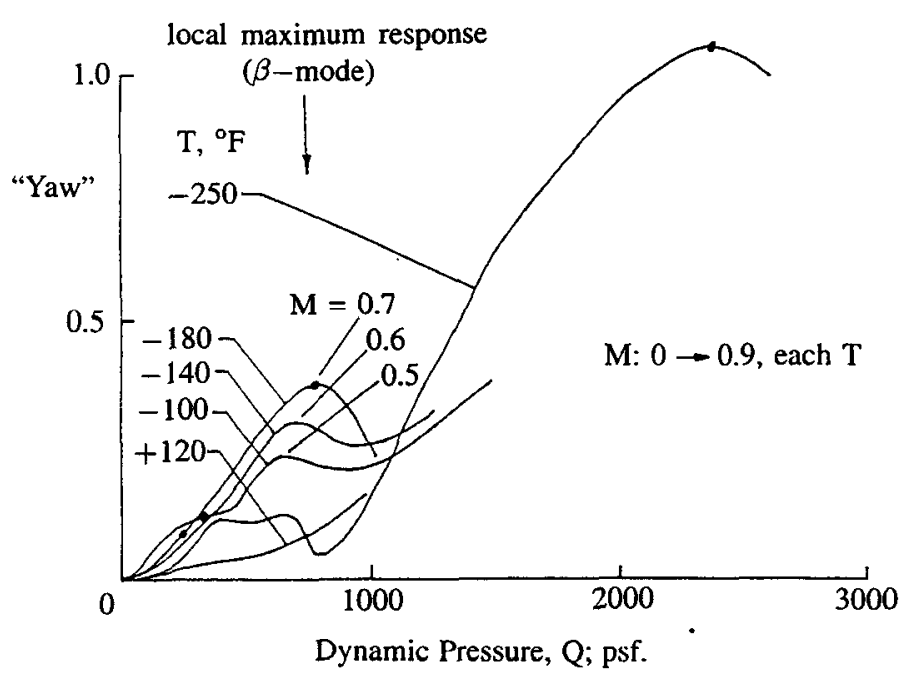

Figure 13 Peak amplitude of "Yaw" spectrum for $f \approx 24-27 \mathrm{~Hz}$. for five temperature-static pressure runs: Mach number varies from 0 to 0.9 for each run.

response with increasing dynamic pressure is seen in Figure 13 and, in addition, there is observed a local maximum response, most pronounced for $\mathrm{T}=-180^{\circ}$ and $\mathrm{M}$ $=0.7$. Noting that the frequency of the $\beta$-mode resonance sweeps through this $25-28 \mathrm{~Hz}$. range of the model "yaw" response, it is evident that this is the mechanism producing the local maximum response motions. It is also apparent that this is not the mechanism responsible for the larger response shown at the highest dynamic pressures. Still, much testing is performed at lower power conditions, and understanding of this loading mechanism might lead to better tunnel performance at those conditions where it can be present.

In summary, the presence of the $\beta$-mode is not sufficient in itself to explain observed model vibrations for the highest power conditions. As the analyses discussed above indicated, for these conditions the frequency of the $\beta$-mode is well below the dominant strut modal frequency, and other excitation mechanisms are necessary to complete the understanding of the situation. 


\section{Concluding Remarks}

Investigations into the causes of model vibrations in the National Transonic Facility have been described. The relationship between wind tunnel resonance, encountered in the unsteady aerodynamic literature, and local aeroacoustic features known as Parker modes is explained. The lowest frequency of these modes, the $\beta$-mode, is shown to interact with structural dynamic modes of the NTF model support structure in aeroelastic analyses. Measurements from the NTF demonstrate the presence of the $\beta$-mode and indicate that a portion of the vibration response of the model support structure, and the attached model, can be attributed to this mechanism.

\section{Acknowledgement}

The author would like to thank Christopher Rumsey, Mark Sanetrik, Robert Biedron, Duane Melson, and Edward Parlette for providing the thin layer Navier-Stokes code calculations shown in Figure 10.

\section{References}

1. Bruce, W. E. Jr.; and Gloss, B. B.: The U. S. National Transonic Facility, NTF, Paper No. 3 in AGARD Report No. 774, Special Course on Advances in Cryogenic Wind Tunnel Technology, NOv. 1989.

2. Igoe, W. B.: Analysi of Fluctuating Static Pressure Measurements in the National Transonic Facility, NASA TP 3475, March 1996.

3. Young, C; Popernack, T.; and Gloss, B: National Transonic Facility Model and Model Support Vibration Problems, AIAA Paper No. 90-1416, 1990.

4. Buehrle, R. D.; Young, C. P., Jr.; Balakrishna, S; and Kilgore, W. A.: Experimental Study of Dynamic Interaction Between Model Support Structure and a High Speed Research Model in the National Transonic Facility, AIAA Paper No. 94-1623, 1994

5. Young, C. P., Jr.; Gergert, D. W.; Butler, T. W.; and Herring, F. M.: Buffet Test in the National Transonic Facility, AIAA Paper No. 92-4032.

6. Strganac, T. W.: A Study of Aeroelastic Stability for the Model Support System of the National Transonic Facility, AIAA Paper No. 88-2033, 1988.
7. Whitlow, W. Jr.; Bennett, R. M.; and Strganac, T. W.: Analysis of Vibrations of the National Transonic Facility Model Support System Using a 3-D Aeroelastic Code, AIAA Paper No. 89-2207, 1989.

8. Runyan, H. L.; Woolston, D. S.; and Rainey, A. G.: Theoretical and Experimental Investigation of the Effect of Tunnel Walls on the Forces on an Oscillating Airfoil in Two-Dimensional Subsonic Compressible Flow, NACA Report 1262, 1956.

9. Parker, R.: Resonance Effects in Wake Shedding From Parallel Plates: Calculation of Resonant Frequencies, J. of Sound and Vibration, Vol 5(2), 1967, pp.330-343.

10. Parker, R.: The Effect of the Acoustic Properties of the Environment on Vibration of a Flat Plate Subject to Direct Excitation and to Excitation by Vortex Shedding in an Airstream, J. of Sound and Vibration, Vol 20(1), 1972, pp. 93-112.

11. Parker, R.; and Stoneman, S. A. T.: The Excitations and Consequences of Acoustic REsonances in Enclosed Fluid Flow Around Solid Bodies, Proc. Instn, Mech, Engrs., Vol. 203, 1989, pp 9-19.

12. Mabey, D. G.; The Resonance Frequencies of Ventilated Wind Tunnels, RAE Technical Report 78038, 1978.

13. Lee, I.: Resonance Prediction for Closed and Open Wind Tunnels by the Finite-Element Method, AIAA J. Vol. 27, No. 4, April 1989, pp. 391-398.

14. Kock, W.: Resonant Acoustic Frequencies of Flat Plate Cascades, J. of Sound and Vibration, Vol 88)2), 1983, pp 233-242.

15. Edwards, J. W.: Transonic Shock Oscillations Calculated with a New Interactive Boundary Layer Coupling Method, AIAA Paper No. 93-0777, 1993.

16. Edwards, J. W.: Transonic Shock Oscillations and Wing Flutter Calculated with an Interactive Boundary Layer Coupling Method, NASA TM 110284, August 1996.

17. Rumsey, C. L.; Sanetrik, M. D.; Biedron, R. T.; Melson, N. D.; and Parlette, E. B.: Efficiency and Accuracy of Time-Accurate Turbulent NavierStokes Computations, AIAA Paper No. 95-1835, 1995. 